Bull. Korean Math. Soc. 52 (2015), No. 5, pp. 1711-1719

http://dx.doi.org/10.4134/BKMS.2015.52.5.1711

\title{
A NOTE OF WEIGHTED COMPOSITION OPERATORS ON BLOCH-TYPE SPACES
}

\author{
SongXiaO Li AND Jizhen Zhou
}

\begin{abstract}
We obtain a new criterion for the boundedness and compactness of the weighted composition operators $\psi C_{\varphi}$ from $\mathcal{B}^{\alpha}(0<\alpha<1)$ to $\mathcal{B}^{\beta}$ in terms of the sequence $\left\{\psi \varphi^{n}\right\}$. An estimate for the essential norm of $\psi C_{\varphi}$ is also given.
\end{abstract}

\section{Introduction}

Denote by $H(\mathbb{D})$ the space of all analytic functions on the unit disk $\mathbb{D}=\{z$ : $|z|<1\}$ in the complex plane. Let $0<\alpha<\infty$. An $f \in H(\mathbb{D})$ is said to belong to Bloch-type spaces(or $\alpha$-Bloch spaces), denoted by $\mathcal{B}^{\alpha}$, if

$$
\|f\|_{\alpha}=\sup _{z \in \mathbb{D}}\left|f^{\prime}(z)\right|\left(1-|z|^{2}\right)^{\alpha}<\infty .
$$

The classical Bloch space $\mathcal{B}$ is just $\mathcal{B}^{1}$. It is clear that $\mathcal{B}^{\alpha}$ is a Banach space with the norm $\|f\|_{\mathcal{B}^{\alpha}}=|f(0)|+\|f\|_{\alpha}$. See $[1,15,16]$ for the theory of Bloch-type spaces.

Let $X$ and $Y$ be Banach spaces of analytic functions on $\mathbb{D}, \psi \in H(\mathbb{D})$ and let $\varphi$ be an analytic self mapping of $\mathbb{D}$. The weighted composition operator with symbols $\psi$ and $\varphi$ from $X$ to $Y$ is the operator $\psi C_{\varphi}$ defined by

$$
\psi C_{\varphi} f=M_{\psi} C_{\varphi} f=\psi(f \circ \varphi) \text { for } f \in X,
$$

where $M_{\psi}$ denotes the multiplication operator with symbol $\psi$ and $C_{\varphi}$ denotes the composition operator with symbol $\varphi$. A basic problem concerning composition operators on various Banach function spaces is to relate the operator theoretic properties of $C_{\varphi}$ to the function theoretic properties of the symbol $\varphi$, which attracted a lots of attention recently, the reader can refer to [3].

Recall that the essential norm of a operator $T$ between $X$ and $Y$ is the distance to the compact operators $K$, that is $\|T\|_{e}^{X \rightarrow Y}=\inf \{\|T-K\|: K$ is compact $\}$, where $\|\cdot\|$ is the operator norm. It is easy to see that $\|T\|_{e}^{X \rightarrow Y}=0$ if and only if $T$ is compact.

Received November 19, 2014.

2010 Mathematics Subject Classification. 47B33, 30H30.

Key words and phrases. weighted composition operator, Bloch-type space, essential norm. 
It is well known that the composition operator is automatically bounded on the Bloch space by Schwarz-Pick Lemma. The compactness of the composition operator on the Bloch space was characterized in [7]. In [13], Wulan, Zheng and Zhu obtained a new characterization for the compactness of the composition operator acting on the Bloch space as follows:

Theorem A. Let $\varphi$ be an analytic self-map of $\mathbb{D}$. Then $C_{\varphi}$ is compact on the Bloch space if and only if

$$
\lim _{n \rightarrow \infty}\left\|\varphi^{n}\right\|_{\mathcal{B}}=0
$$

The boundedness and the compactness of composition operators on Bloch type spaces was given in [5] by Lou. In [14], Zhao extended Theorem A to Bloch-type spaces. Among other results, he proved the following result.

Theorem B. Let $0<\alpha, \beta<\infty$, and $\varphi$ be a self-map of $\mathbb{D}$. Then the essential norm of composition operator $C_{\varphi}: \mathcal{B}^{\alpha} \rightarrow \mathcal{B}^{\beta}$ is

$$
\left\|C_{\varphi}\right\|_{e}^{\mathcal{B}^{\alpha} \rightarrow \mathcal{B}^{\beta}}=\left(\frac{e}{2 \alpha}\right)^{\alpha} \limsup _{n \rightarrow \infty} n^{\alpha-1}\left\|\varphi^{n}\right\|_{\beta} .
$$

In [10], Ohno, Stroethoff and Zhao studied the boundedness and compactness of weighted composition operators on Bloch-type spaces. The essential norm of weighted composition operators on Bloch-type spaces are given in [6]. In [8], Manhas and Zhao gave an estimate for the essential norm of $\psi C_{\varphi}: \mathcal{B}^{\alpha} \rightarrow \mathcal{B}^{\beta}$. Especially, when $0<\alpha<1$, they obtained the following result.

Theorem C. Suppose $0<\alpha<1$ and $0<\beta<\infty$ and suppose that $\psi C_{\varphi}: \mathcal{B}^{\alpha} \rightarrow$ $\mathcal{B}^{\beta}$ is bounded. Then the essential norm of composition operator $C_{\varphi}: \mathcal{B}^{\alpha} \rightarrow \mathcal{B}^{\beta}$ is

$$
\left\|\psi C_{\varphi}\right\|_{e}^{\mathcal{B}^{\alpha} \rightarrow \mathcal{B}^{\beta}}=\left(\frac{e}{2 \alpha}\right)^{\alpha} \limsup _{n \rightarrow \infty} n^{\alpha-1}\left\|I_{\psi}\left(\varphi^{n}\right)\right\|_{\beta}
$$

where

$$
I_{\psi} f(z)=\int_{0}^{z} f^{\prime}(\zeta) \psi(\zeta) d \zeta
$$

Motivated by Theorems A, B and C, in this work we show that $\psi C_{\varphi}: \mathcal{B}^{\alpha} \rightarrow$ $\mathcal{B}^{\beta}$ is bounded (respectively, compact) if and only if the sequence $\left(\frac{\left\|\psi \varphi^{n}\right\|_{\beta}}{\left\|z^{n}\right\|_{\alpha}}\right)_{n=1}^{\infty}$ is bounded (respectively, convergent to 0 as $n \rightarrow \infty$ ) when $0<\alpha<1$. Moreover, we give the exact essential norm for the operators $\psi C_{\varphi}$.

\section{Boundedness of $\psi C_{\varphi}$ on Bloch-type spaces}

In this section, we give a characterization for the boundedness of $\psi C_{\varphi}$ : $\mathcal{B}^{\alpha} \rightarrow \mathcal{B}^{\beta}$ when $0<\alpha<1$. For this purpose, we need the following result which is given in [10]. 
Lemma 2.1. Let $0<\alpha<1,0<\beta<\infty, \psi \in H(\mathbb{D})$ and let $\varphi$ be an analytic self-map of $\mathbb{D}$. Then $\psi C_{\varphi}: \mathcal{B}^{\alpha} \rightarrow \mathcal{B}^{\beta}$ is bounded if and only if $\psi \in \mathcal{B}^{\beta}$ and

$$
\sup _{z \in \mathbb{D}}|\psi(z)| \frac{\left(1-|z|^{2}\right)^{\beta}}{\left(1-|\varphi(z)|^{2}\right)^{\alpha}}\left|\varphi^{\prime}(z)\right|<\infty .
$$

The boundedness of $\psi C_{\varphi}: \mathcal{B}^{\alpha} \rightarrow \mathcal{B}^{\beta}$ implies that $\psi \in \mathcal{B}^{\beta}$ if we choose $f=1 \in \mathcal{B}^{\alpha}$. So we always assume that $\psi \in \mathcal{B}^{\beta}$. We are now ready to state and prove the main results in this section.

Theorem 2.2. Let $0<\alpha<1,0<\beta<\infty, \psi \in H(\mathbb{D})$ and let $\varphi$ be an analytic self-map of $\mathbb{D}$. Assume that $\psi \in \mathcal{B}^{\beta}$, then $\psi C_{\varphi}: \mathcal{B}^{\alpha} \rightarrow \mathcal{B}^{\beta}$ is bounded if and only if

$$
\sup _{n \in \mathbb{N}} \frac{\left\|\psi \varphi^{n}\right\|_{\beta}}{\left\|z^{n}\right\|_{\alpha}}<\infty .
$$

Proof. Assume that $\psi C_{\varphi}: \mathcal{B}^{\alpha} \rightarrow \mathcal{B}^{\beta}$ is bounded. Since for any nonnegative integer $n$, the sequence $f_{n}(z)=z^{n} /\left\|z^{n}\right\|_{\alpha}$ is bounded in $\mathcal{B}^{\alpha}$, we get

$$
\frac{\left\|\psi \varphi^{n}\right\|_{\beta}}{\left\|z^{n}\right\|_{\alpha}}=\left\|\psi C_{\varphi}\left(\frac{z^{n}}{\left\|z^{n}\right\|_{\alpha}}\right)\right\|_{\beta} \leq\left\|\psi C_{\varphi}\right\|_{\mathcal{B}^{\alpha} \rightarrow \mathcal{B}^{\beta}}<\infty .
$$

The desired result follows.

Now we assume that (1) holds. Let $M:=\sup _{n \in \mathbb{N}} \frac{\left\|\psi \varphi^{n}\right\|_{\beta}}{\left\|z^{n}\right\|_{\alpha}}$. For $n \geq 2$, we define

$$
\mathbb{D}_{n}=\left\{z \in \mathbb{D}: r_{n} \leq|\varphi(z)| \leq r_{n+1}\right\},
$$

where $r_{n}=\sqrt{\frac{n-1}{n-1+2 \alpha}}$. Fix an integer $N>2$. For $|\varphi(z)| \leq r_{N}$, by the product rule, we have

$$
\begin{aligned}
\frac{\left(1-|z|^{2}\right)^{\beta}\left|\psi(z) \varphi^{\prime}(z)\right|}{\left(1-|\varphi(z)|^{2}\right)^{\alpha}} & \leq \frac{\left(1-|z|^{2}\right)^{\beta}\left(\left|(\psi \varphi)^{\prime}(z)\right|+\left|\psi^{\prime}(z) \varphi(z)\right|\right)}{\left(1-r_{N}^{2}\right)^{\alpha}} \\
& \leq\left(\frac{N-1+2 \alpha}{2 \alpha}\right)^{\alpha}\left(\|\psi \varphi\|_{\beta}+\|\psi\|_{\beta}\right)<\infty .
\end{aligned}
$$

Note that for $n \in \mathbb{N}$ and $0<\alpha<1$,

$\left\|z^{n}\right\|_{\alpha}=\left\|z^{n}\right\|_{\mathcal{B}^{\alpha}}=\max _{z \in \mathbb{D}} n\left|z^{n-1}\right|\left(1-|z|^{2}\right)^{\alpha}$

$$
=n\left(\frac{2 \alpha}{n-1+2 \alpha}\right)^{\alpha}\left(\frac{n-1}{n-1+2 \alpha}\right)^{(n-1) / 2}=n\left(1-r_{n}^{2}\right)^{\alpha} r_{n}^{n-1},
$$

the max is attained at any point on the circle with radius $r_{n}$.

For $|\varphi(z)|>r_{N}$, there exists $n \geq N$ such that $z \in \mathbb{D}_{n}$. So

$$
\begin{aligned}
\frac{\left(1-|z|^{2}\right)^{\beta}\left|\psi(z) \varphi^{\prime}(z)\right|}{\left(1-|\varphi(z)|^{2}\right)^{\alpha}} & =\frac{\left(1-|z|^{2}\right)^{\beta}\left|\psi(z) \varphi(z)^{n-1} \varphi^{\prime}(z)\right|\left\|z^{n}\right\|_{\alpha}}{\left(1-|\varphi(z)|^{2}\right)^{\alpha}|\varphi(z)|^{n-1}\left\|z^{n}\right\|_{\alpha}} \\
& \leq \frac{\left(1-|z|^{2}\right)^{\beta}\left|\psi(z) \varphi(z)^{n-1} \varphi^{\prime}(z)\right| n\left(1-r_{n}^{2}\right)^{\alpha} r_{n}^{n-1}}{\left(1-r_{n+1}^{2}\right)^{\alpha} r_{n}^{n-1}\left\|z^{n}\right\|_{\alpha}}
\end{aligned}
$$




$$
\begin{aligned}
& \leq 2^{\alpha} \frac{\left(1-|z|^{2}\right)^{\beta}\left[\left|\left(\psi \varphi^{n}\right)^{\prime}(z)\right|+\left|\psi^{\prime}(z) \varphi^{n}(z)\right|\right]}{\left\|z^{n}\right\|_{\alpha}} \\
& \leq 2^{\alpha} \frac{\left\|\psi \varphi^{n}\right\|_{\beta}+\|\psi\|_{\beta}}{\left\|z^{n}\right\|_{\alpha}} \leq 2^{\alpha}\left(M+\frac{\|\psi\|_{\beta}}{\left\|z^{n}\right\|_{\alpha}}\right)<\infty,
\end{aligned}
$$

where we apply the inequality $1-r_{n}^{2}<2\left(1-r_{n+1}^{2}\right), n \geq 2$ and the fact that $\left\|z^{n}\right\|_{\alpha} \rightarrow \infty$ as $n \rightarrow \infty$ when $0<\alpha<1$. From (2), (4) and Lemma 2.1, we deduce that $\psi C_{\varphi}$ is bounded from $\mathcal{B}^{\alpha}$ into $\mathcal{B}^{\beta}$.

Let $\psi \equiv 1$. We get the following result, which appeared in Theorem 2.1 of [14].

Corollary 2.3. Let $0<\alpha<1,0<\beta<\infty$ and let $\varphi$ be an analytic self-map of $\mathbb{D}$. The $C_{\varphi}: \mathcal{B}^{\alpha} \rightarrow \mathcal{B}^{\beta}$ is bounded if and only if

$$
\sup _{n \in \mathbb{N}} \frac{\left\|\varphi^{n}\right\|_{\beta}}{\left\|z^{n}\right\|_{\alpha}}<\infty \text {. }
$$

\section{Essential norm of $\psi C_{\varphi}$ on Bloch-type spaces}

The following criterion for compactness follows by a standard argument similar, for example, to that outlined in Proposition 3.11 of [3].

Lemma 3.1. Let $0<\alpha<1,0<\beta<\infty, \psi \in H(\mathbb{D})$ and let $\varphi$ be an analytic self-map of $\mathbb{D}$. The operator $\psi C_{\varphi}: \mathcal{B}^{\alpha} \rightarrow \mathcal{B}^{\beta}$ is compact if and only if for any bounded sequence $\left\{f_{n}\right\}_{n \in \mathbb{N}}$ in $\mathcal{B}^{\alpha}$ which converges to zero uniformly on compact subsets of $\mathbb{D}$, we have $\left\|\psi C_{\varphi} f_{n}\right\|_{\mathcal{B}^{\beta}} \rightarrow 0$ as $n \rightarrow \infty$.

Denote by $K_{r} f(z)=f(r z)$ for $r \in(0,1)$. Then $K_{r}$ is a compact operator on the space $\mathcal{B}^{\alpha}$ for $\alpha>0$. It is easy to see that $\left\|K_{r}\right\| \leq 1$. Let $I$ denote the identity operator. The following result can be found in [6].

Lemma 3.2. Let $0<\alpha<1$. There is a sequence $\left\{r_{k}\right\}$, with $0<r_{k}<1$ tending to 1 , such that the compact operator $L_{n}=\frac{1}{n} \sum_{k=1}^{n} K_{r_{k}}$ on $\mathcal{B}_{0}^{\alpha}$ satisfies

(i) For any $t \in(0,1), \lim _{n \rightarrow \infty} \sup _{\|f\|_{\mathcal{B}^{\alpha}} \leq 1} \sup _{|z| \leq t}\left|\left(\left(I-L_{n}\right) f\right)^{\prime}(z)\right|=0$.

(ii) $\lim _{n \rightarrow \infty} \sup _{\|f\|_{\mathcal{B}^{\alpha}<1} \leq 1} \sup _{z \in \mathbb{D}}\left|\left(I-L_{n}\right) f(z)\right|=0$.

(iii) $\limsup _{n \rightarrow \infty}\left\|I-L_{n}\right\| \leq 1$.

Furthermore, these statements holds as well for the sequence of biadjoints $L_{n}^{* *}$ (which is the same form as $L_{n}$ ) on $\mathcal{B}^{\alpha}$.

Theorem 3.3. Let $0<\alpha<1,0<\beta<\infty, \psi \in H(\mathbb{D})$ and let $\varphi$ be an analytic self-map of $\mathbb{D}$. Suppose that $\psi C_{\varphi}: \mathcal{B}^{\alpha} \rightarrow \mathcal{B}^{\beta}$ is bounded, then the essential norm of $\psi C_{\varphi}: \mathcal{B}^{\alpha} \rightarrow \mathcal{B}^{\beta}$ is

$$
\left\|\psi C_{\varphi}\right\|_{e}^{\mathcal{B}^{\alpha} \rightarrow \mathcal{B}^{\beta}}=\limsup _{n \rightarrow \infty} \frac{\left\|\psi \varphi^{n}\right\|_{\beta}}{\left\|z^{n}\right\|_{\alpha}} .
$$


Proof. By the assumption that $\psi C_{\varphi}$ is bounded from $\mathcal{B}^{\alpha}$ into $\mathcal{B}^{\beta}$, we easily get that $\|\psi \varphi\|_{\beta}<\infty$ and $\|\psi\|_{\beta}<\infty$. Moreover, $\sup _{z \in \mathbb{D}}\left(1-|z|^{2}\right)^{\beta}\left|\psi(z) \varphi^{\prime}(z)\right|<\infty$.

First, we prove that (5) holds when $\sup _{z \in \mathbb{D}}|\varphi(z)|<1$. Assume $\sup _{z \in \mathbb{D}}|\varphi(z)|$ $<1$, then there is a number $\delta \in(0,1)$ such that $\sup _{z \in \mathbb{D}}|\varphi(z)|<\delta$. Choose a bounded sequence $\left\{f_{n}\right\}_{n \in \mathbb{N}}$ in $\mathcal{B}^{\alpha}$ which converges to zero uniformly on compact subset of $\mathbb{D}$. Then $\left\{f_{n}^{\prime}\right\}_{n \in \mathbb{N}}$ also converges to zero on compact subsets of $\mathbb{D}$ as $n \rightarrow \infty$. It follows that

$$
\begin{aligned}
\lim _{n \rightarrow \infty}\left\|\psi C_{\varphi} f_{n}\right\|_{\mathcal{B}^{\beta}} & =\lim _{n \rightarrow \infty}\left(\left|\psi(0) f_{n}(\varphi(0))\right|+\left\|\psi C_{\varphi} f_{n}\right\|_{\beta}\right) \\
& =\lim _{n \rightarrow \infty} \sup _{z \in \mathbb{D}}\left|\psi^{\prime}(z) f_{n}(\varphi(z))+\psi(z) f_{n}^{\prime}(\varphi(z)) \varphi^{\prime}(z)\right|\left(1-|z|^{2}\right)^{\beta} \\
& \leq \lim _{n \rightarrow \infty}\left(\|\psi\|_{\beta} \sup _{|z| \leq \delta}\left|f_{n}(z)\right|+\sup _{|z| \leq \delta}\left|f_{n}^{\prime}(z)\right| \sup _{z \in \mathbb{D}}\left|\psi \varphi^{\prime}\right|\left(1-|z|^{2}\right)^{\beta}\right) \\
& =0 .
\end{aligned}
$$

Then the operator $\psi C_{\varphi}: \mathcal{B}^{\alpha} \rightarrow \mathcal{B}^{\beta}$ is compact by Lemma 3.1. This gives that $\left\|\psi C_{\varphi}\right\|_{e}^{\mathcal{B}^{\alpha} \rightarrow \mathcal{B}^{\beta}}=0$. On the other hand,

$$
\begin{aligned}
& \limsup _{n \rightarrow \infty} \frac{\left\|\psi \varphi^{n}\right\|_{\beta}}{\left\|z^{n}\right\|_{\alpha}} \\
= & \limsup _{n \rightarrow \infty} \sup _{z \in \mathbb{D}} \frac{\left(1-|z|^{2}\right)^{\beta}\left|\psi^{\prime}(z) \varphi^{n}(z)+n \psi(z) \varphi^{n-1}(z) \varphi^{\prime}(z)\right|}{\left\|z^{n}\right\|_{\alpha}} \\
\leq & \limsup _{n \rightarrow \infty} \sup _{z \in \mathbb{D}} \frac{\left(1-|z|^{2}\right)^{\beta}\left(\left|\psi^{\prime}(z) \varphi^{n}(z)\right|+n\left|\psi(z) \varphi^{n-1}(z) \varphi^{\prime}(z)\right|\right)}{\left\|z^{n}\right\|_{\alpha}} \\
\leq & \limsup _{n \rightarrow \infty}\left(\frac{\|\psi\|_{\beta} \delta^{n}}{\left\|z^{n}\right\|_{\alpha}}+\sup _{z \in \mathbb{D}} \frac{n\left|(\psi \varphi)^{\prime}(z)-\psi^{\prime}(z) \varphi(z)\right| \delta^{n-1}}{\left\|z^{n}\right\|_{\alpha}}\left(1-|z|^{2}\right)^{\beta}\right) \\
\leq & \limsup _{n \rightarrow \infty} \frac{\|\psi\|_{\beta} \delta^{n}+n\|\psi\|_{\beta} \delta^{n}+n\|\psi \varphi\|_{\beta} \delta^{n-1}}{\left\|z^{n}\right\|_{\alpha}}=0 .
\end{aligned}
$$

Thus, when $\sup _{z \in \mathbb{D}}|\varphi(z)|<1$, we have

$$
\left\|\psi C_{\varphi}\right\|_{e}^{\mathcal{B}^{\alpha} \rightarrow \mathcal{B}^{\beta}}=\limsup _{n \rightarrow \infty} \frac{\left\|\psi \varphi^{n}\right\|_{\beta}}{\left\|z^{n}\right\|_{\alpha}}=0 .
$$

Next, we prove that (5) holds when $\sup _{z \in \mathbb{D}}|\varphi(z)|=1$.

We first give the lower estimate for the essential norm. Choose the sequence of function $f_{n}(z)=z^{n} /\left\|z^{n}\right\|_{\alpha}, n \in \mathbb{N}$. Then $\left\|f_{n}\right\|_{\mathcal{B}^{\alpha}}=1$, and $f_{n}$ converges to zero weakly on $\mathcal{B}^{\alpha}$ as $n \rightarrow \infty$. Thus we have $\lim _{n \rightarrow \infty}\left\|K f_{n}\right\|_{\mathcal{B}^{\alpha}}=0$ for any given compact operator $K$ on $\mathcal{B}^{\alpha}$. The basic inequality gives that

$$
\left\|\psi C_{\varphi}-K\right\|^{\mathcal{B}^{\alpha} \rightarrow \mathcal{B}^{\beta}} \geq\left\|\left(\psi C_{\varphi}-K\right) f_{n}\right\|_{\mathcal{B}^{\beta}} \geq\left\|\psi C_{\varphi} f_{n}\right\|_{\mathcal{B}^{\beta}}-\left\|K f_{n}\right\|_{\mathcal{B}^{\beta}} .
$$

Thus we obtain that

$$
\left\|\psi C_{\varphi}-K\right\|^{\mathcal{B}^{\alpha} \rightarrow \mathcal{B}^{\beta}} \geq \limsup _{n \rightarrow \infty}\left\|\psi C_{\varphi} f_{n}\right\|_{\mathcal{B}^{\beta}} \geq \limsup _{n \rightarrow \infty}\left\|\psi C_{\varphi} f_{n}\right\|_{\beta}
$$


So we have

$$
\left\|\psi C_{\varphi}\right\|_{e}^{\mathcal{B}^{\alpha} \rightarrow \mathcal{B}^{\beta}}=\inf _{K}\left\|\psi C_{\varphi}-K\right\| \geq \limsup _{n \rightarrow \infty} \frac{\left\|\psi \varphi^{n}\right\|_{\beta}}{\left\|z^{n}\right\|_{\alpha}} .
$$

Next, still under the assumption that $\sup _{z \in \mathbb{D}}|\varphi(z)|=1$, we give the upper estimate for the essential norm. Let $L_{n}$ be the sequence of operators given in Lemma 3.2. Since $L_{n}$ is compact on $\mathcal{B}^{\alpha}$ and $\psi C_{\varphi}: \mathcal{B}^{\alpha} \rightarrow \mathcal{B}^{\beta}$ is bounded, then $\psi C_{\varphi} L_{n}: \mathcal{B}^{\alpha} \rightarrow \mathcal{B}^{\beta}$ is also compact. Hence

$$
\begin{aligned}
\left\|\psi C_{\varphi}\right\|_{e}^{\mathcal{B}^{\alpha} \rightarrow \mathcal{B}^{\beta}} & \leq \limsup _{n \rightarrow \infty}\left\|\psi C_{\varphi}-\psi C_{\varphi} L_{n}\right\|_{\mathcal{B}^{\alpha} \rightarrow \mathcal{B}^{\beta}} \\
& =\limsup _{n \rightarrow \infty}\left\|\psi C_{\varphi}\left(I-L_{n}\right)\right\|_{\mathcal{B}^{\alpha} \rightarrow \mathcal{B}^{\beta}} \\
& =\limsup _{n \rightarrow \infty} \sup _{\|f\|_{\mathcal{B}^{\alpha} \leq 1}\left\|\psi C_{\varphi}\left(I-L_{n}\right) f\right\|_{\mathcal{B}^{\beta}}} \\
& =I_{1}+I_{2},
\end{aligned}
$$

where

$$
I_{1}=\limsup _{n \rightarrow \infty} \sup _{\|f\|_{\mathcal{B}^{\alpha} \leq 1}}\left|\psi(0)\left(\left(I-L_{n}\right) f\right)(\varphi(0))\right|
$$

and

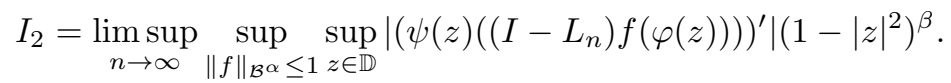

It follows from Lemma 3.2 that $I_{1}=0$.

Let $k$ be the smallest positive integer such that $\mathbb{D}_{k} \neq \emptyset$. Since $\sup _{z \in \mathbb{D}}|\varphi(z)|=$ $1, \mathbb{D}_{n}$ is not empty for every integer $n \geq k$ and $\mathbb{D}=\bigcup_{n=k}^{\infty} \mathbb{D}_{n}$. Then we have that $I_{2} \leq I_{21}+I_{22}+I_{23}$, where

$$
\begin{aligned}
& I_{21}=\limsup _{n \rightarrow \infty} \sup _{\|f\|_{\mathcal{B}^{\alpha} \leq 1}} \sup _{z \in \mathbb{D}}\left|\psi^{\prime}(z)\right|\left|\left(\left(I-L_{n}\right) f\right)(\varphi(z))\right|\left(1-|z|^{2}\right)^{\beta}, \\
& I_{22}=\limsup _{n \rightarrow \infty} \sup _{\|f\|_{\mathcal{B}^{\alpha}} \leq 1} \sup _{N \leq i} \sup _{z \in \mathbb{D}_{i}}\left|\psi(z)\left(\left(I-L_{n}\right) f\right)^{\prime}(\varphi(z)) \varphi^{\prime}(z)\right|\left(1-|z|^{2}\right)^{\beta}
\end{aligned}
$$

and

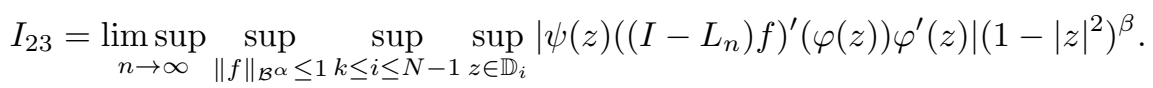

Here $N$ is a positive integer determined as follows.

From (3), for any given $\epsilon>0$, there exists a positive integer $N$ such that

$$
\frac{\|\psi\|_{\beta}}{\left\|z^{n}\right\|_{\mathcal{B}^{\alpha}}}<\epsilon \text { and } \frac{\left(1-r_{n}^{2}\right)^{\alpha}}{\left(1-r_{n+1}^{2}\right)^{\alpha}}<1+\epsilon,
$$

when $n \geq N$.

For such $N$ it follows by Lemma 3.2 that

$$
\begin{aligned}
& I_{22}=\limsup _{n \rightarrow \infty} \sup _{\|f\|_{\mathcal{B}^{\alpha} \leq 1}} \sup _{N \leq i} \sup _{z \in \mathbb{D}_{i}}\left|\psi(z)\left(\left(I-L_{n}\right) f\right)^{\prime}(\varphi(z)) \varphi^{\prime}(z)\right|\left(1-|z|^{2}\right)^{\beta} \\
& \leq \limsup _{n \rightarrow \infty} \sup _{\|f\|_{\mathcal{B}} \leq 1}\left\|\left(I-L_{n}\right) f\right\|_{\alpha} \sup _{N \leq i} \sup _{z \in \mathbb{D}_{i}} \frac{\left|\psi(z) \varphi^{\prime}(z)\right|}{\left(1-|\varphi(z)|^{2}\right)^{\alpha}}\left(1-|z|^{2}\right)^{\beta}
\end{aligned}
$$




$$
\begin{aligned}
& \leq \limsup _{n \rightarrow \infty}\left\|I-L_{n}\right\| \sup _{N \leq i \in \sup _{i}} \frac{\left|\psi(z) \varphi^{\prime}(z) \varphi^{i-1}(z)\right|\left\|z^{i}\right\|_{\alpha}}{\left(1-|\varphi(z)|^{2}\right)^{\alpha}|\varphi(z)|^{i-1}\left\|z^{i}\right\|_{\alpha}}\left(1-|z|^{2}\right)^{\beta} \\
& \leq \sup _{N \leq i} \sup _{z \in \mathbb{D}_{i}} \frac{i\left(1-r_{i}^{2}\right)^{\alpha} r_{i}^{i-1}\left|\psi(z) \varphi^{\prime}(z) \varphi^{i-1}(z)\right|}{\left(1-r_{i+1}^{2}\right)^{\alpha} r_{i}^{i-1}\left\|z^{i}\right\|_{\alpha}}\left(1-|z|^{2}\right)^{\beta} \\
& \leq(1+\epsilon) \sup _{N \leq i} \sup _{z \in \mathbb{D}_{i}} \frac{\left|\left(\psi \varphi^{i}\right)^{\prime}(z)\right|+\left|\psi^{\prime}(z) \varphi^{i}(z)\right|}{\left\|z^{i}\right\|_{\alpha}}\left(1-|z|^{2}\right)^{\beta} \\
& \leq(1+\epsilon) \sup _{N \leq i} \frac{\left\|\psi \varphi^{i}\right\|_{\beta}+\|\psi\|_{\beta}}{\left\|z^{i}\right\|_{\alpha}} \\
& \leq(1+\epsilon)\left(\sup _{N \leq i} \frac{\left\|\psi \varphi^{i}\right\|_{\beta}}{\left\|z^{i}\right\|_{\alpha}}+\epsilon\right) .
\end{aligned}
$$

Also it follows by Lemma 3.2 that

$$
\begin{aligned}
& I_{23}=\limsup _{n \rightarrow \infty} \sup _{\|f\|_{\mathcal{B}^{\alpha} \leq 1}} \sup _{k \leq i \leq N-1} \sup _{z \in \mathbb{D}_{i}}\left|\psi(z)\left(\left(I-L_{n}\right) f\right)^{\prime}(\varphi(z)) \varphi^{\prime}(z)\right|\left(1-|z|^{2}\right)^{\beta}
\end{aligned}
$$

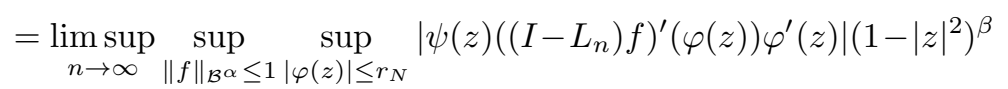

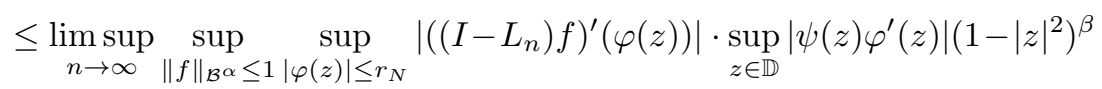

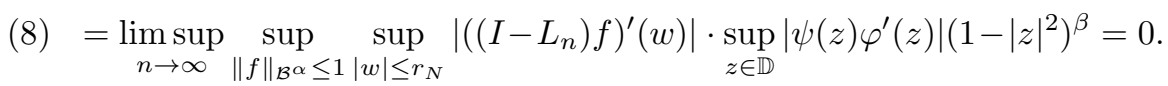

By Lemma 3.2, we have

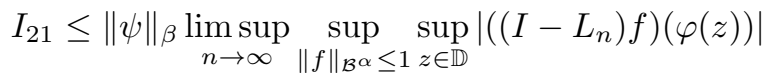

$$
\begin{aligned}
& \leq\|\psi\|_{\beta} \limsup _{n \rightarrow \infty} \sup _{\|f\|_{\mathcal{B}^{\alpha} \leq 1} \sup _{w \in \mathbb{D}}\left|\left(\left(I-L_{n}\right) f\right)(w)\right|=0 .}
\end{aligned}
$$

It follows from (7), (8) and (9) that

$$
I_{2}<(1+\epsilon)\left(\sup _{N \leq i} \frac{\left\|\psi \varphi^{i}\right\|_{\beta}}{\left\|z^{i}\right\|_{\alpha}}+\epsilon\right)
$$

From (10) we obtain that

$$
\left\|\psi C_{\varphi}\right\|_{e}^{\mathcal{B}^{\alpha} \rightarrow \mathcal{B}^{\beta}} \leq I_{1}+I_{2}<(1+\epsilon)\left(\sup _{N \leq i} \frac{\left\|\psi \varphi^{i}\right\|_{\beta}}{\left\|z^{i}\right\|_{\alpha}}+\epsilon\right) .
$$

Since $\epsilon$ is arbitrary, it follows that

$$
\left\|\psi C_{\varphi}\right\|_{e}^{\mathcal{B}^{\alpha} \rightarrow \mathcal{B}^{\beta}} \leq \limsup _{i \rightarrow \infty} \frac{\left\|\psi \varphi^{i}\right\|_{\beta}}{\left\|z^{i}\right\|_{\alpha}} .
$$

The proof is complete.

From Theorem 3.3, we obtain the following result. 
Corollary 3.4. Let $0<\alpha<1,0<\beta<\infty, \psi \in H(\mathbb{D})$ and let $\varphi$ be an analytic self-map of $\mathbb{D}$. Suppose that $\psi \in \mathcal{B}^{\beta}$. Then $\psi C_{\varphi}: \mathcal{B}^{\alpha} \rightarrow \mathcal{B}^{\beta}$ is compact if and only if

$$
\limsup _{n \rightarrow \infty} \frac{\left\|\psi \varphi^{n}\right\|_{\beta}}{\left\|z^{n}\right\|_{\alpha}}=0 .
$$

Corollary 3.5. Let $0<\alpha<1,0<\beta<\infty$ and let $\varphi$ be an analytic self-map of $\mathbb{D}$. Then the essential norm of $C_{\varphi}: \mathcal{B}^{\alpha} \rightarrow \mathcal{B}^{\beta}$ is

$$
\left\|C_{\varphi}\right\|_{e}^{\mathcal{B}^{\alpha} \rightarrow \mathcal{B}^{\beta}}=\limsup _{n \rightarrow \infty} \frac{\left\|\varphi^{n}\right\|_{\beta}}{\left\|z^{n}\right\|_{\alpha}} .
$$

Acknowledgment. This project was partially supported by the Macao Science and Technology Development Fund (No.083/2014/A2).

\section{References}

[1] J. Anderson, J. Clunie, and Ch. Pommerenke, On Bloch functions and normal functions, J. Reine Angew. Math. 270 (1974), 12-37.

[2] M. Contreras and A. Hernandez-Diaz, Weighted composition operators in weighted Banach spaces of analytic functions, J. Austral. Math. Soc. Ser. A 69 (2000), no. 1, 41-60.

[3] C. C. Cowen and B. D. MacCluer, Composition Operators on Spaces of Analytic Functions, Studies in Advanced Mathematics, CRC Press, Boca Raton, 1995.

[4] S. Li and S. Stević, Weighted composition operators from Bergman-type spaces into Bloch spaces, Proc. Indian Acad. Sci. Math. Sci. 117 (2007), no. 3, 371-385.

[5] Z. Lou, Composition operators on Bloch type spaces, Analysis 23 (2003), no. 1, 81-95.

[6] B. Maccluer and R. Zhao, Essential norm of weighted composition operators between Bloch-type spaces, Rocky Mountain J. Math. 33 (2003), no. 4, 1437-1458.

[7] K. Madigan and A. Matheson, Compact composition operators on the Bloch space, Trans. Amer. Math. Soc. 347 (1995), no. 7, 2679-2687.

[8] J. Manhas and R. Zhao, New estimates of essential norms of weighted composition operators between Bloch type spaces, J. Math. Anal. Appl. 389 (2012), no. 1, 32-47.

[9] A. Montes-Rodriguez, Weighted composition operators on weighted Banach spaces of analytic functions, J. London Math. Soc. 61 (2000), no. 3, 872-884.

[10] S. Ohno, K. Stroethoff, and R. Zhao, Weighted composition operators between Bloch-type spaces, Rocky Mountain J. Math. 33 (2003), no. 1, 191-215.

[11] S. Ohno and R. Zhao, Weighted composition operators on the Bloch space, Bull. Austral. Math. Soc. 63 (2001), no. 2, 177-185.

[12] S. Stević and R. Agarwal, Weighted composition operators from logarithmic Bloch-type spaces to Bloch-type spaces, J. Inequal. Appl. 2009 (2009), Art. ID 964814, 21 pp.

[13] H. Wulan, D. Zheng, and K. Zhu, Compact composition operators on BMOA and the Bloch space, Proc. Amer. Math. Soc. 137 (2009), no. 11, 3861-3868.

[14] R. Zhao, Essential norms of composition operators between Bloch type spaces, Proc. Amer. Math. Soc. 138 (2010), no. 7, 2537-2546.

[15] K. Zhu, Bloch type spaces of analytic functions, Rocky Mountain J. Math. 23 (1993), no. $3,1143-1177$.

[16] _ Operator Theory in Function Spaces, Second edition, American Mathematical Society, Providence, 2007. 
SONGXIAO Li

Institute of System EngineERING

Macau University of Science and Technology

Avenida Wai Long, TAIPA, Macau

E-mail address: jyulsx@163.com

JIZHEN ZHOU

SCHOOL OF SCIENCE

Anhui University of Science And Technology

232001, Huainan, Anhui, P. R. China

E-mail address: hope189@163.com 